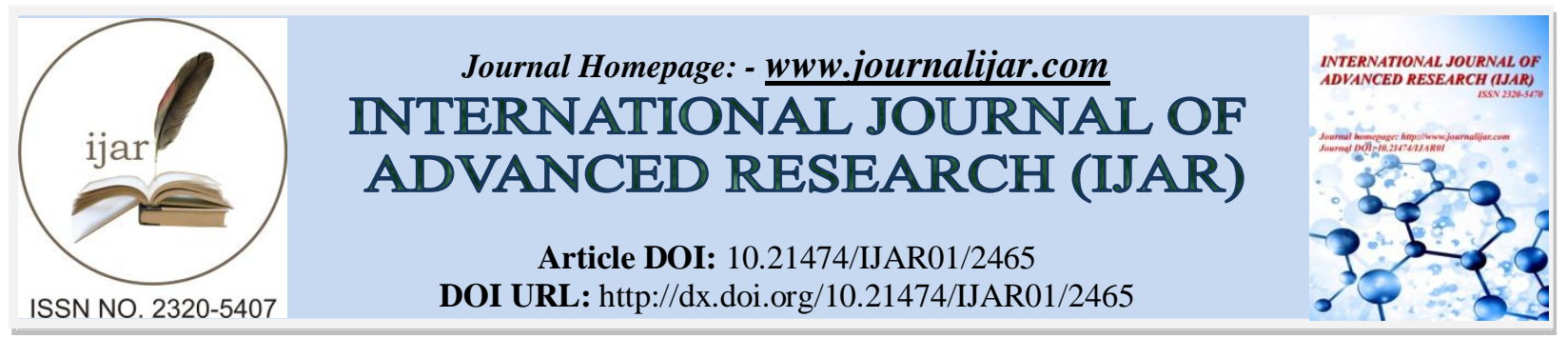

RESEARCH ARTICLE

\title{
INFLUENCE OF PRASEODYMIUM ON THE OPTICAL, STRUCTURAL AND DIELECTRIC PROPERTIES OF KDP CRYSTALS GROWN BY SEED ROTATION TECHNIQUE.
}

\section{Roopa $\mathbf{V}^{1^{*}}$ and $\mathrm{R}$.Ananda Kumari ${ }^{2}$.}

1. Department of Physics, Sree Siddaganga College of Arts, Science and Commerce for Women, Tumkur 572102, India.

2. Department of Physics, Sree Siddaganga College of Arts, Science and Commerce, Tumkur 572102, India.

\section{Manuscript Info}

\section{Manuscript History}

Received: 23 October 2016

Final Accepted: 21 November 2016

Published: December 2016

Key words:-

Crystal growth;

Seed Rotation;

Optical properties;

Dielectric properties.

\section{Abstract}

The Praseodymium doped potassium dihydrogen orthophosphate (KDP) crystals were grown from aqueous solution by the temperature lowering method using a microcontroller based seed rotation technique and slow evaporation technique with the vision to improve the properties of the crystal. The chemical composition of the grown crystals is confirmed by EDAX Analysis. The grown crystal was characterized by PXRD analysis confirm the crystalline nature and shifts in peak positions due to doping is observed. Using Scherer's equation particle size has been calculated. Solubility of crystals grown by slow evaporation technique is determined. The solubility curve shows that praseodymium doped KDP crystals has higher solubility than the pure KDP. The SHG efficiency is determined by Kurtz powder technique. It is found that relative SHG conversion efficiency of crystal grown by Seed rotation technique is greater compared to other technique. The UV-Vis spectrum shows that percentage transmission of crystals grown by seed rotation technique is greater compared to slow evaporation. The electronic band transition is studied from the plot of $(\alpha h v)^{2}$ versus photon energy (hv) and the band gap energy has been calculated. The dielectric constant, dielectric loss and ac conductivity of the crystal were studied as a function of frequency and the results are discussed. The functional groups are identified by FT-Raman analysis. The Optical transmission and SHG studies shows the suitability of the ingot for optical applications.

Copy Right, IJAR, 2016,. All rights reserved.

\section{Introduction:-}

Advanced research on efficient nonlinear optical material (NLO) is intensively studied for various optical device applications. Potassium dihydrogen orthophosphate (KDP) $\mathrm{KH}_{2} \mathrm{PO}_{4}$ is a best known NLO material and has been used for second harmonic generation for high pulse energy, laser frequency conversion, low repetition $(<100 \mathrm{~Hz})$ rate lasers, electro-optical modulation and Q-switching applications [1-3]. As a result, significant efforts have been made to find novel and efficient NLO materials.

Corresponding Author:- Roopa V.

Address:- Department of Physics, Sree Siddaganga College for Women, Tumkur-572102 
The study of the crystallization behavior of KDP and the factors influencing its structural properties is still of great interest. The most important factor which influences the growth rate, the surface morphology of crystal is impurities $[4,5]$. An impurity can suppress, enhance or stop the growth of crystal completely. Modern technical tasks like high power laser systems have a great demand for very large size crystals. The use of special additives is an effective way to accelerate the growth rate. The beneficial effect of additives on the growth process and properties of crystals has been applied in recent years [6-8].

The most efficient additives are reagents with metal ions that have the same properties as that of bulk solutions which can change the properties of solution such as viscosity, surface tension, etc. without deteriorating the optical qualities of crystals. Hence Praseodymium is selected as an additive in the KDP solution and doped KDP crystals were grown from the aqueous solution with seed rotation and slow evaporation technique and the grown crystals are subjected to different characterizations like powder X-ray diffraction, FT-Raman analysis, optical transmission, dielectric study, EDAX and second harmonic generation efficiency (SHG) studies.

\section{Experiments:- \\ Solubility studies:-}

KDP (AR) grade, Praseodymium from Merck and triple distilled water were used for all the studies. The solubility studies were done for pure KDP and KDP doped with Praseodymium grown by slow evaporation method. Solubility studies were carried out in a constant temperature water bath (CTB). Stirring was achieved using an immersible magnetic stirrer. The solution was stirred continuously for 5 hours to achieve stabilization. Solubility was determined by gravimetric analysis for different temperatures $\left(25-50^{\circ} \mathrm{C}\right)$. The solubility curve of pure KDP and KDP doped Praseodymium is shown in Fig. 1. It is observed from the solubility curve that the solubility of KDP doped Praseodymium increases with increase in the molar weight of KDP and has positive temperature co-efficient.

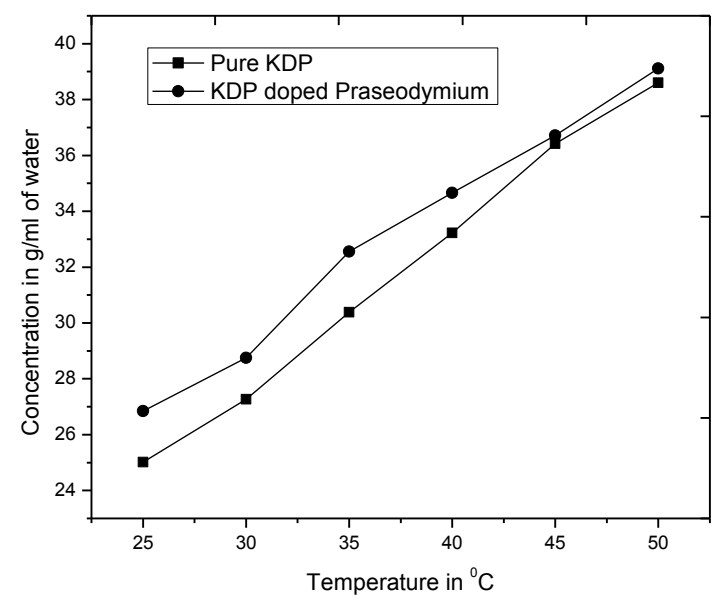

Fig. 1:- Solubility curve of pure and Praseodymium-doped KDP crystals.

\section{Crystal growth:-}

All the growth processes were carried out in a constant temperature bath with an accuracy of $70{ }^{\circ} \mathrm{C}$. Stirring the solution reduces the natural convection-induced temperature oscillations by homogenizing the bulk solution. Hence, the importance of optimum rates of rotation in crystal growth processes has gained recognition in the past decade [9]. The two most widely used stirring mechanisms are the rotation of the seed and/or the rotation of the crystallizer. In the present work, KDP crystals doped with Praseodymium were grown from aqueous solution by continuously rotating the growing crystal at $40 \mathrm{rpm}$. This apparatus consists of a seed rotation controller coupled with a stepper motor which is controlled by using a microcontroller based drive. This controller rotates the seed holder in the crystallizer. The seed crystals mounted on the center of the platform made up of acrylic material and is fixed into the crystallizer. The seed mount platform mix the solution very well and makes the solution more stable, which resulted in better crystal quality. The aqueous solutions at the saturation temperature $40^{\circ} \mathrm{C}$ was filtered in a closed system to remove extraneous solid and colloidal particles. Then the solution was overheated at $50^{\circ} \mathrm{c}$ for one 
day, to make the solution stable against spontaneous nucleation under a high super saturation [10]. After overheating, the temperature of the solution was reduced slightly above the saturation point and seed crystals were mounted on the platform. From the saturation point, the temperature was decreased at $0.1^{\circ} \mathrm{C}$ per day at the beginning of the growth. As the growth of the crystal progressed, the temperature rate was decreased. After reaching the room temperature, crystals was harvested. The grown crystals by seed rotation technique and slow evaporation method are shown in Fig. 2.
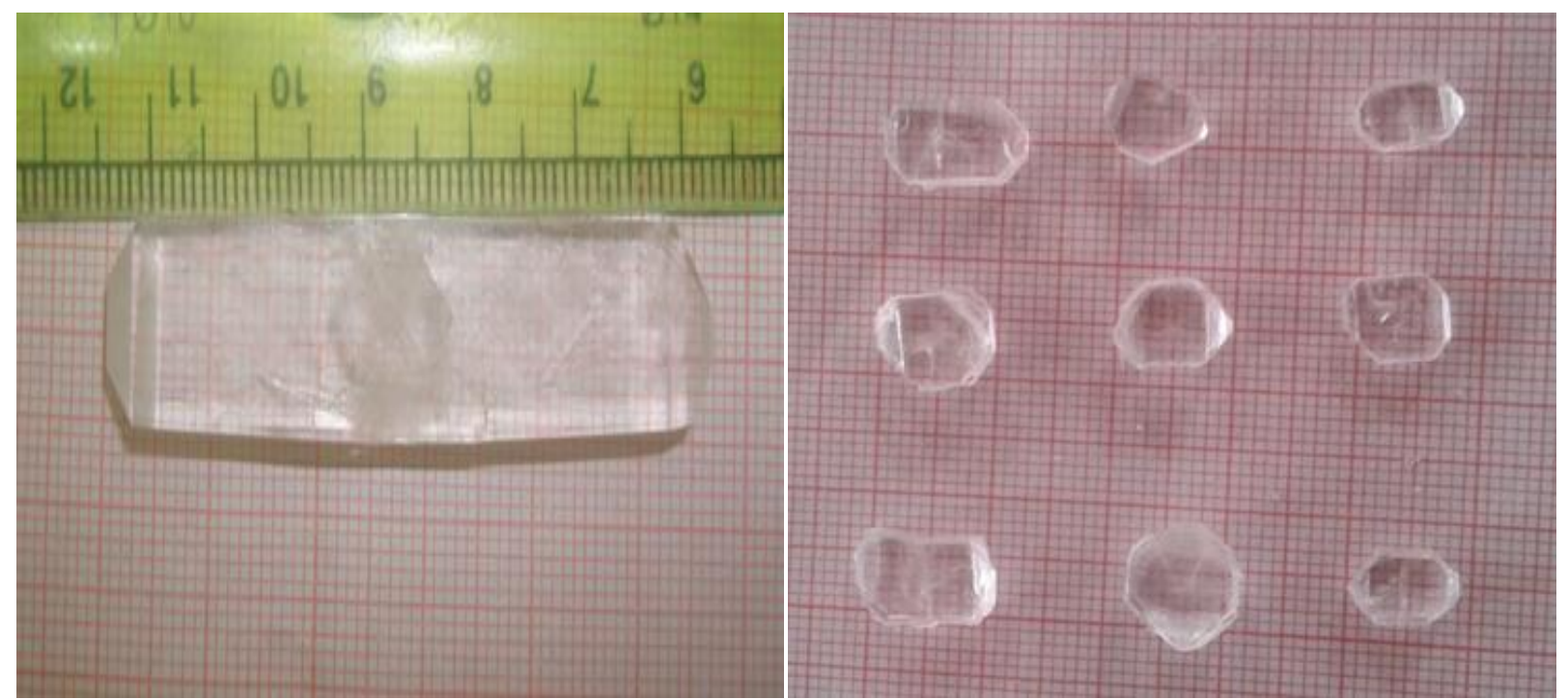

Fig. 2:- Photograph of Praseodymium-doped KDP crystals by grown Seed Rotation technique and slow Evaporation technique

\section{Results and Discussion:- EDAX Analysis:-}

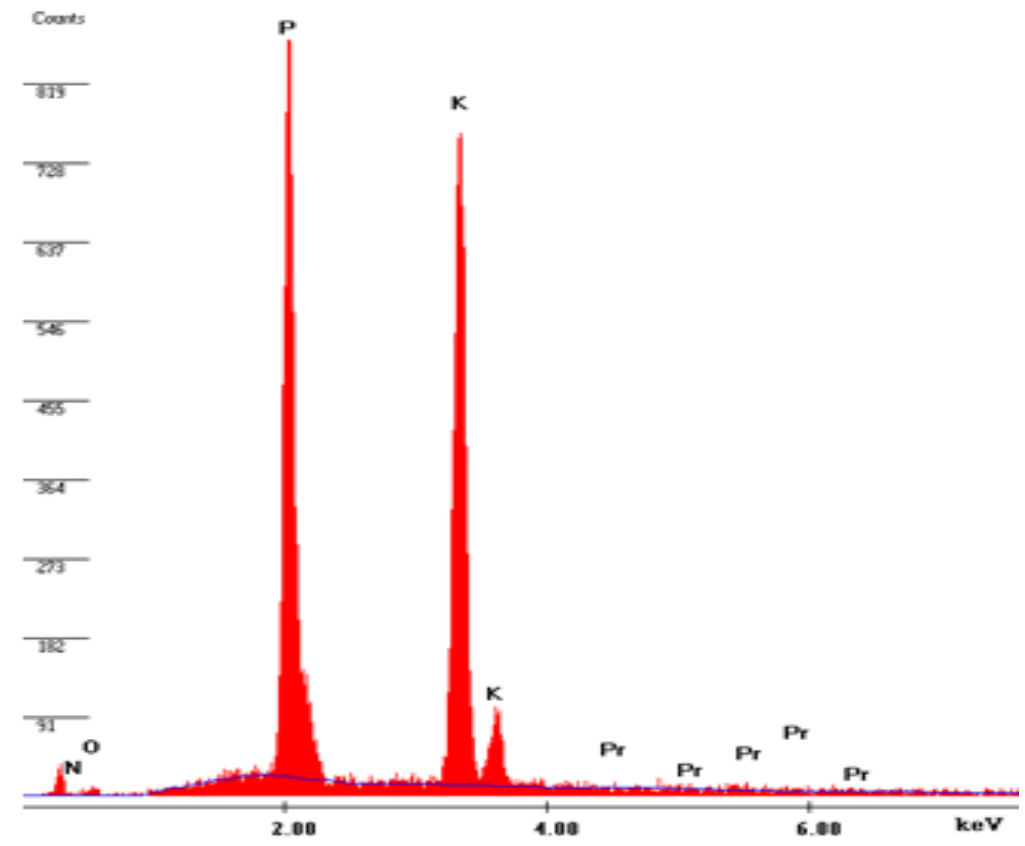

Fig. 3:- EDAX Spectrum of Praseodymium-doped KDP crystals(Slow Evaporation)

In order to confirm the presence of the Praseodymium, the grown crystals was subjected to EDAX (Energy dispersive X-ray analysis). The EDAX spectra for KDP doped Praseodymium grown by Slow evaporation and Seed Rotation technique was recorded and analyzed. The spectrum shows the peaks of potassium, phosphate, oxygen, nitrate and Praseodymium suggesting that the Praseodymium dopant has entered into the crystal lattice of KDP. The 
recorded spectrum for the grown crystals are shown in Fig. $3 \& 4$. The Observed weight percentage of elements in the doped KDP crystal are given in the Table $1 \& 2$.

Table 1:- Shows the estimated Weight \% of KDP doped Praseodymium Crystal (Slow Evaporation method)

\begin{tabular}{|c|c|c|}
\hline Element & Weight \% & Atom \% \\
\hline $\mathrm{N}$ & 1.85 & 4.51 \\
\hline $\mathrm{O}$ & 2.26 & 4.83 \\
\hline $\mathrm{P}$ & 37.22 & 41.04 \\
\hline $\mathrm{K}$ & 56.11 & 49.01 \\
\hline $\mathrm{Pr}$ & 2.56 & 0.62 \\
\hline Total & 100.00 & 100.00 \\
\hline
\end{tabular}

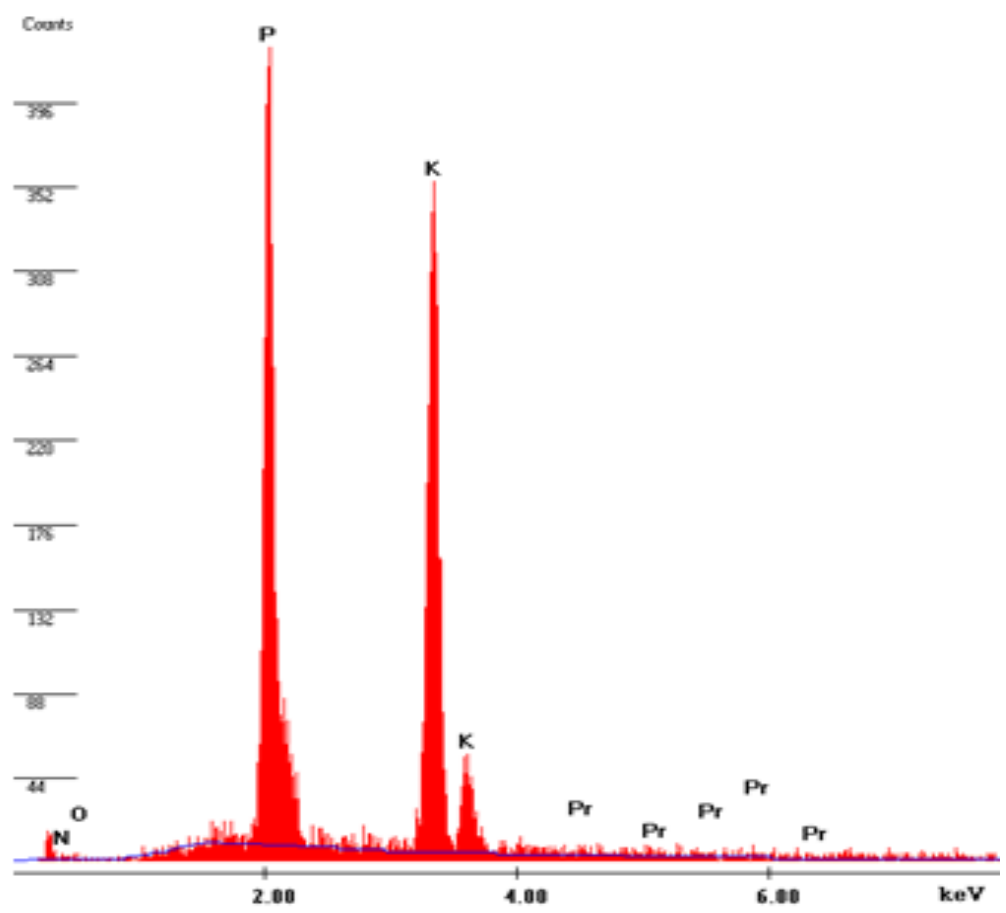

Fig. 4:-EDAX Spectrum of Praseodymium-doped KDP crystals(Seed Rotation)

Table 2:- Shows the estimated Weight \% of KDP doped Praseodymium Crystal (Seed Rotation method)

\begin{tabular}{|l|l|l|}
\hline Element & Weight \% & Atom \% \\
\hline $\mathrm{N}$ & 2.07 & 5.16 \\
\hline $\mathrm{O}$ & 0.84 & 1.83 \\
\hline $\mathrm{P}$ & 38.26 & 43.14 \\
\hline $\mathrm{K}$ & 54.70 & 48.85 \\
\hline $\mathrm{Pr}$ & 4.13 & 1.02 \\
\hline Total & 100.00 & 100.00 \\
\hline
\end{tabular}

\section{Powder X-ray diffraction studies:-}

Powder X-ray diffraction studies was performed on grown crystals to identify the phase formation and degree of crystal perfection. X-ray powder patterns of grown crystals was recorded using XrdwinPD 4-dectris computer based diffractometer with a characteristic $\mathrm{Cu} \mathrm{K \alpha}(1.540598)$ radiations from $10^{\circ}$ to $60^{\circ}$ at a scan rate of $10^{\circ} / \mathrm{min}$. The Xrd pattern of the grown crystals are shown in Fig. 5. The occurrence of sharp peaks at specific Bragg's angle shows the crystallinity of the grown crystals. It is clear from the pattern that the entry of the dopant in the modified composition of KDP crystal lead to a change in the intensity of peaks when compared to the peaks of pure KDP crystals. Average crystalline size (D) was estimated using the following relation: 
$D=\frac{0.9 \lambda}{\beta \cos \theta}$

where $\lambda$ is wavelength of the X-ray radiation, $\beta$ is full width at half maximum (FWHM) of diffraction peak (in rad), and $\theta$ is scattering angle. Further, the dislocation density $(\delta)$, Stacking fault (SF), and micro strain $(\varepsilon)$ was estimated by the relation:

$$
\begin{aligned}
& \delta=\frac{1}{D^{2}} \\
& \varepsilon=\frac{\beta \cos \theta}{4} \\
& \mathrm{SF}=\frac{2 \pi^{2}}{\sqrt{\tan \theta}}
\end{aligned}
$$

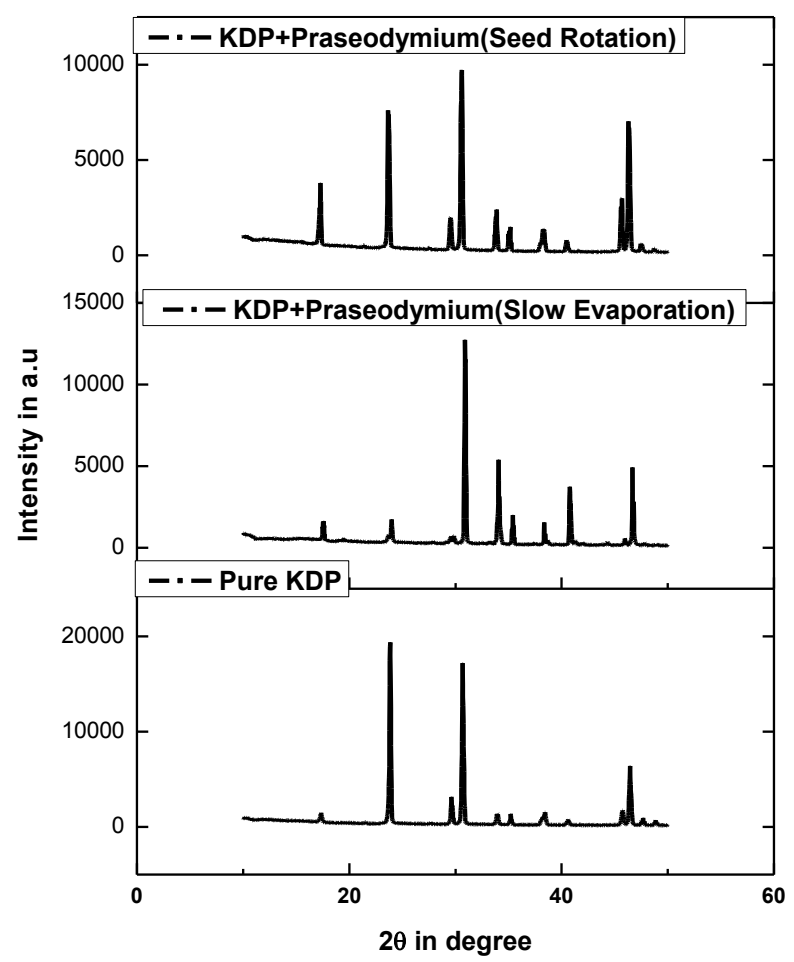

Fig. 5:- Xrd patterns of pure and Praseodymium-doped KDP crystals.

The obtained structural parameters were given in Table 3.Williamson and Hall (W-H) plots was used to estimate the micro strain in KDP doped Praseodymium crystal grown by different methods using the relation

$\beta \cos \theta=\frac{k \lambda}{D}+4 \varepsilon \sin \theta$

where $\varepsilon$ is strain associated with the crystal. Equation (5) represents a straight line between $4 \sin \theta(\mathrm{X}$-axis) and $\beta \cos \theta$ (Y-axis). The slope of line gives the strain $(\varepsilon)$ and intercept $(\mathrm{k} \lambda / \mathrm{D})$ of this line on $\mathrm{Y}$-axis gives grain size (D). Fig. 6. shows the W-H plots of grown crystals and the estimated strain values was shown in Table 3. 


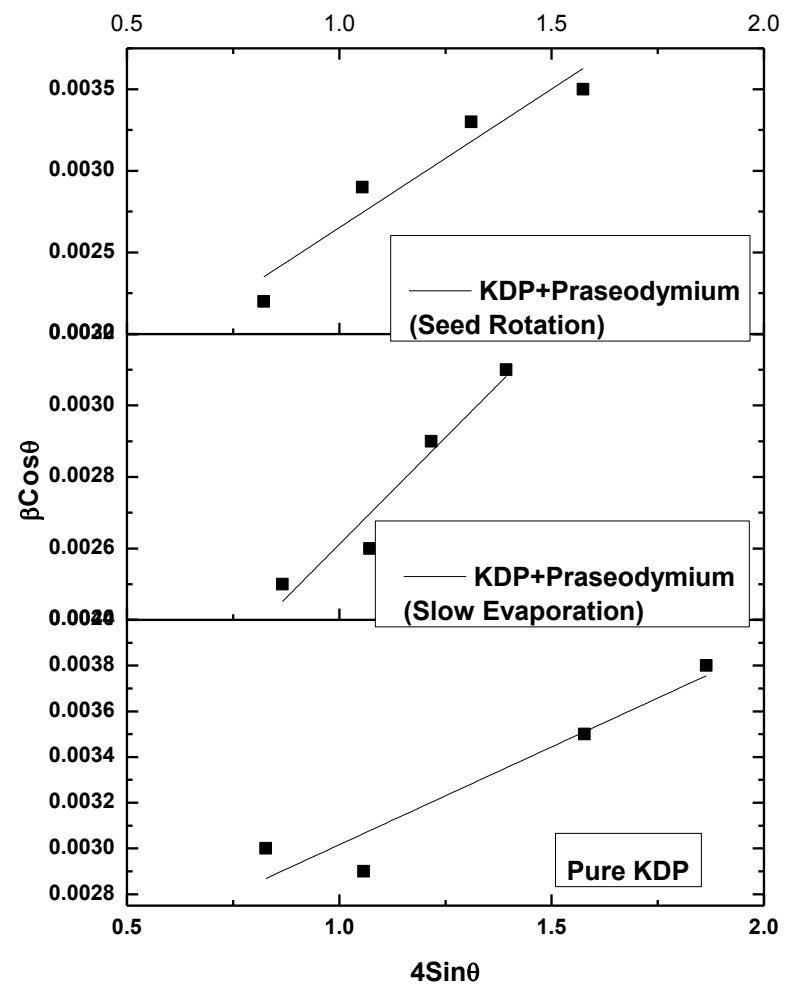

Fig. 6:- W-H Plot of grown crystals

Table 3:- Estimated crystalline size, strain, dislocation density and surface factor of grown crystals.

\begin{tabular}{|l|c|c|c|c|c|}
\hline \multirow{2}{*}{ Sample } & \multicolumn{2}{|c|}{ Crystalline size (nm) } & Strain & $\begin{array}{c}\text { Stacking } \\
\text { fault(SF) }\end{array}$ & $\begin{array}{c}\text { Dislocation density } \\
\mathbf{\delta}\left(\mathbf{1 0} \mathbf{1 0}^{\mathbf{1 4}} \mathbf{m}^{-\mathbf{2}}\right.\end{array}$ \\
\cline { 2 - 5 } & Scherer's formula & W-H plot & & & \\
\hline & & & & 17.39 & 5.15 \\
\hline Pure KDP & 44.03 & 64.24 & $8.554 \mathrm{E}^{-4}$ & 1.01 \\
\hline $\begin{array}{l}\text { KDP+Pr (Slow } \\
\text { Evaporation) }\end{array}$ & 49.90 & 112.81 & $1.28 \mathrm{E}^{-3}$ & 17.35 & 4.46 \\
\hline $\begin{array}{l}\text { KDP+Pr (Seed } \\
\text { Rotation) }\end{array}$ & 47.16 & 88.38 & $1.24 \mathrm{E}^{-3}$ & 17.42 & \\
\hline
\end{tabular}

FT-Raman Analysis:-

The Raman spectra of grown crystals were recorded for (101) planes at room temperature in the wave number range of 200 to $2000 \mathrm{~cm}-1$. Raman spectra in the range of 200 to $2000 \mathrm{~cm}-1$ is shown in Fig. 7. The spectra of pure, Praseodymium doped KDP single crystal grown by different methods contain the internal modes of vibrations $[11,12]$ of $\mathrm{H}_{2} \mathrm{PO}_{4}^{-}$in $\mathrm{KH}_{2} \mathrm{PO}_{4}$ at $911 \mathrm{~cm}^{-1}(\mathrm{v} 1), 529 \mathrm{~cm}^{-1}$ (v2), $478 \mathrm{~cm}^{-1}$ (v3), $387 \mathrm{~cm}^{-1}$ (v4) and $351 \mathrm{~cm}^{-1}$ (v5). The peaks with very small intensity at around $1366 \mathrm{~cm}^{-1}, 1527 \mathrm{~cm}^{-1}$ and $1643 \mathrm{~cm}^{-1}$ are corresponding to the lattice vibrations of crystals through the absorption or emission of optical phonons. It is clearly observed from the figure that doping of Praseodymium did not influence the internal vibrational modes of crystals as there is no shift or broadness of the main peak at $911 \mathrm{~cm}^{-1}$ corresponding to the asymmetric stretching vibration of $\mathrm{H}_{2} \mathrm{PO}_{4}{ }^{-}$anions at all concentrations. The same nature of all spectra confirms no deviation in tetragonal phase and also reveals the absence of any additional phase with Praseodymium doping. The Phonon life time $(\tau)$ can be deduced from the Raman scattering by using energy time uncertainty relation:

$\frac{1}{\tau}=\frac{\Delta E}{h / 2 \pi}=2 \pi \Gamma$ 
where $\Delta \mathrm{E}$ is the uncertainty in the energy of the phonon mode, $\mathrm{h} / 2 \pi$ is the Planck constant, and $\Gamma$ is the FWHM of the Raman modes in $\mathrm{cm}^{-1}$. The phonon life time is a combination of two characteristic decay time such as an harmonic decay of the phonon into two or more phonons so that energy and momentum are conserved $\left(\tau_{\mathrm{A}}\right)$ and perturbation of translational symmetry of the crystals due to the presence of impurities, defects and isotropic fluctuations $\left(\tau_{1}\right)$. So the calculated phonon life time is an addition of two characteristic decay time. $\left(\frac{1}{\tau}=\frac{1}{\tau a}+\frac{1}{\tau 1}\right)$. The calculated phonon life time values are listed in Table 4.

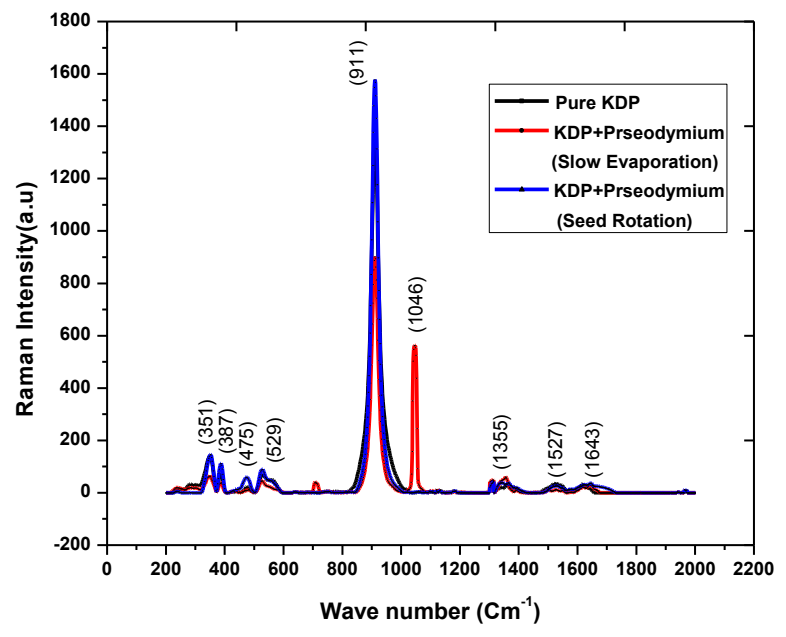

Fig. 7:- FT-Raman Spectrum of grown crystals

Table 4:- Phonon life time of grown crystals

\begin{tabular}{|c|c|c|c|c|c|c|}
\hline \multirow{2}{*}{$\begin{array}{c}\text { Reference } \\
\left(\mathbf{C m}^{-1}\right)\end{array}$} & \multicolumn{2}{|c|}{ Pure KDP } & \multicolumn{2}{|c|}{ KDP+Pr (Slow Evaporation) } & \multicolumn{2}{|c|}{ KDP+ Pr (Seed Rotation) } \\
\hline & $\underset{\substack{\left.\mathbf{C m}^{-1}\right) \\
\text { FWHM }}}{(\Gamma \text { in }}$ & $\begin{array}{c}\text { Phonon life } \\
(\mu \mathrm{s})\end{array}$ & $\underset{\substack{\left.\mathrm{Cm}^{-1}\right) \\
\text { FWHM }(\Gamma \text { in }}}{ }$ & $\begin{array}{c}\text { Phonon life } \\
(\mu \mathrm{s})\end{array}$ & $\begin{array}{c}\text { FWHM ( } \Gamma \text { in } \\
\left.\text { Cm }^{-1}\right)\end{array}$ & $\begin{array}{c}\text { Phonon life } \\
(\mu \mathrm{s})\end{array}$ \\
\hline 351 & 31.42 & 50.65 & 25.63 & 62.09 & 28.86 & 55.14 \\
\hline 387 & 16.43 & 96.86 & 16.67 & 95.47 & 17.64 & 90.22 \\
\hline 529 & 56.95 & 27.94 & 51.42 & 30.95 & 25.11 & 63.38 \\
\hline 911 & 45.99 & 34.60 & 30.98 & 51.37 & 28.94 & 54.99 \\
\hline 1046 & - & - & 15.79 & 10.07 & - & - \\
\hline 1527 & 55.74 & 28.53 & 54.65 & 29.12 & 55.83 & 28.50 \\
\hline 1643 & 37.28 & 42.69 & 68.17 & 23.34 & 24.96 & 63.76 \\
\hline
\end{tabular}

UV-Visible Transmission:-

Crystal plates of pure KDP and KDP doped Praseodymium crystals were cut and polished without any coating for optical measurements. The thickness of the crystals were around $1 \mathrm{~mm}$. Optical transmission spectra were recorded for the crystals in the wavelength region $200-1100 \mathrm{~nm}$ using Perkin-Elmer Lambda $35 \mathrm{UV}$-Vis spectrometer. The recorded UV-Vis spectrum is shown in the Fig. 8. Good optical transmittance and lower cut off wavelength are very essential properties for nonlinear optical (NLO) crystals [13]. It is observed from the figure that the Pure KDP shows $45 \%$ of transmittance, KDP doped praseodymium (Slow Evaporation method) shows $65 \%$ and KDP doped praseodymium (Seed Rotation method) shows $70 \%$ of transmittance. The large transmission in the entire visible region enables it to be a good material for electro-optic and NLO applications. The above results indicate that the addition of praseodymium to pure KDP increased the transmittance. There is a non linear trend in transmittance between 400 to $800 \mathrm{~nm}$ wavelength shows that the light is only transmitted and not absorbed in this visible region. The plot of $(\alpha \mathrm{hv})^{2}$ versus photon energy hv is plotted as shown in Fig. 9. In order to find the value of Eg we make use of the relation :

$\alpha=2.303 \log (\mathrm{T} / \mathrm{d})$

$\alpha$ is absorption coefficient, $d$ is the thickness of the sample and T is the transmittance. $h v$ is the photon energy. 
The values of Eg have been found by taking the intercept of the curve, at which it increases linearly. The wide optical band gap of pure KDP is found to be $4.2 \mathrm{eV}$, and for KDP doped praseodymium crystals is $5.0 \mathrm{eV}$ respectively suggests its suitability for optoelectronics applications.

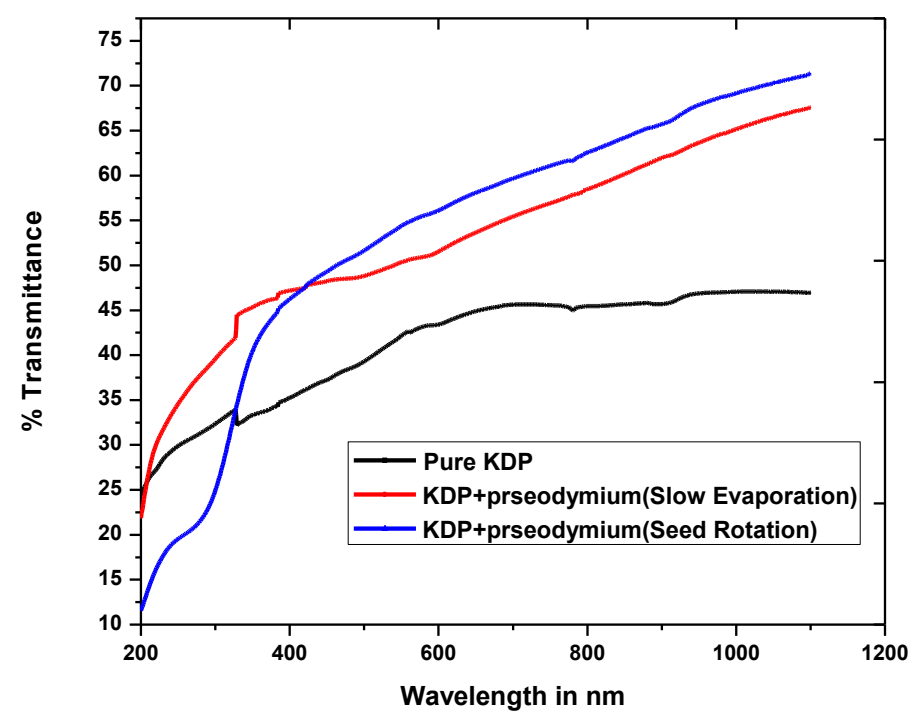

Fig. 8:- UV-Visible Spectrum of grown crystals.

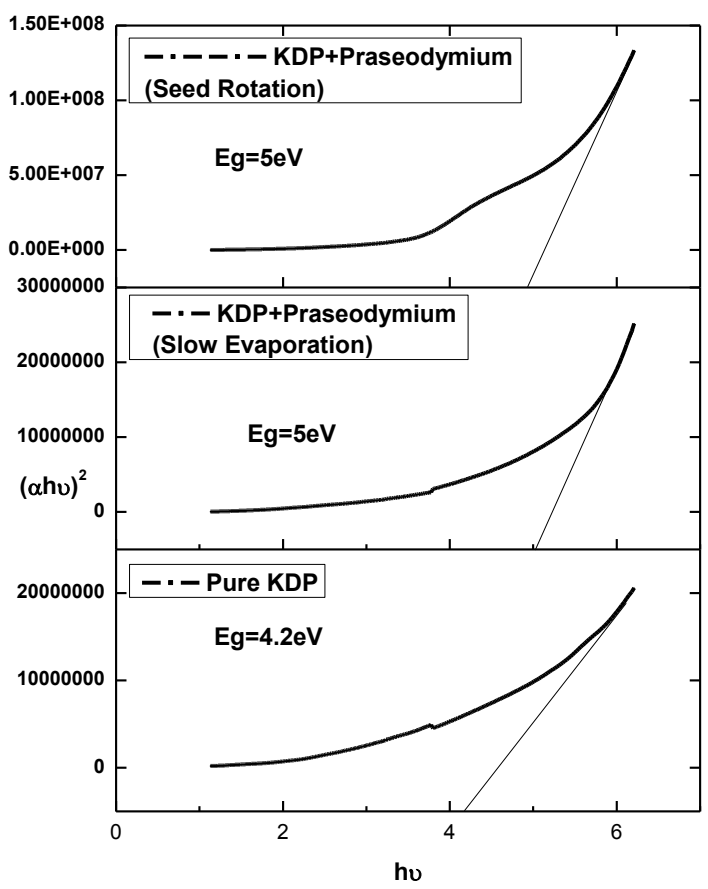

Dielectric Studies:-

Fig. 9:- $(\alpha h v)^{2}$ versus photon energy hv of the grown crystals.

Suitably cut and polished crystals section of pure and praseodymium doped KDP crystal grown by different methods was electroded on either side with air-drying silver paste so that it behaved like a parallel plate capacitor. A 4275A, 
multi frequency LCR meter (Hewlett-Packard) was used to measure capacitance (C) and dissipation factor (D) of the sample as a function of frequency. The dielectric constant $(\varepsilon)$ and dielectric loss $(\tan \delta)$ were calculated from C and D using the relations :

$$
\begin{aligned}
& \varepsilon=\frac{C d}{A \varepsilon 0} \\
& \tan \delta=D \varepsilon
\end{aligned}
$$

where $\mathrm{C}$ is the capacitance of the sample, $\mathrm{d}$ the thickness of the sample, $\mathrm{A}$ is the area of the face in contact with the electrode and $\varepsilon_{0}$ the permittivity of free space.

The variations of dielectric constant $(\varepsilon)$ and dielectric loss $(\tan \delta)$ at room temperature for pure KDP and praseodymium doped KDP crystal grown by different methods are shown in Fig. 10 and 11. It is observed that the dielectric constant $(\varepsilon)$ decreases with the increase in the frequency. The dielectric constant of a material is generally composed of four types of contributions, viz ionic, electronic, orientational and space charge polarizations. At low frequencies all polarizabilities are operative hence $\varepsilon$ is high.

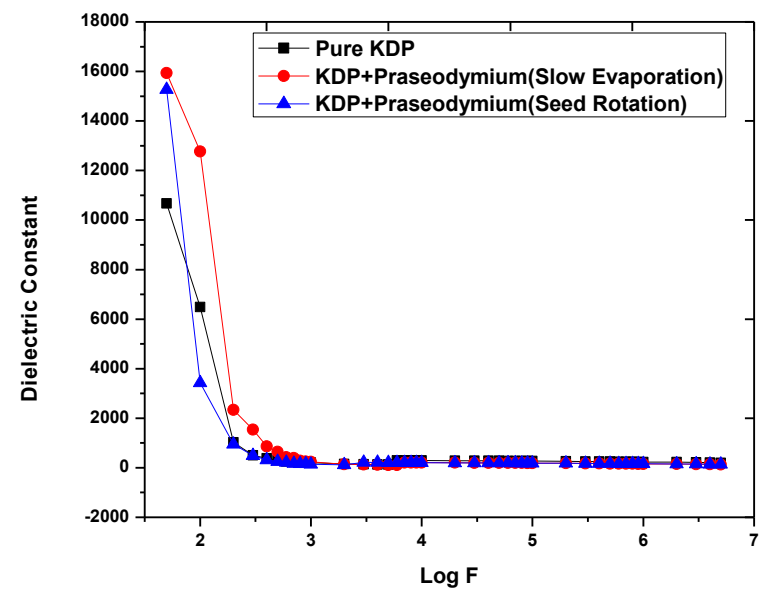

Fig. 10:- Variation of dielectric constant with log frequency for grown crystals.

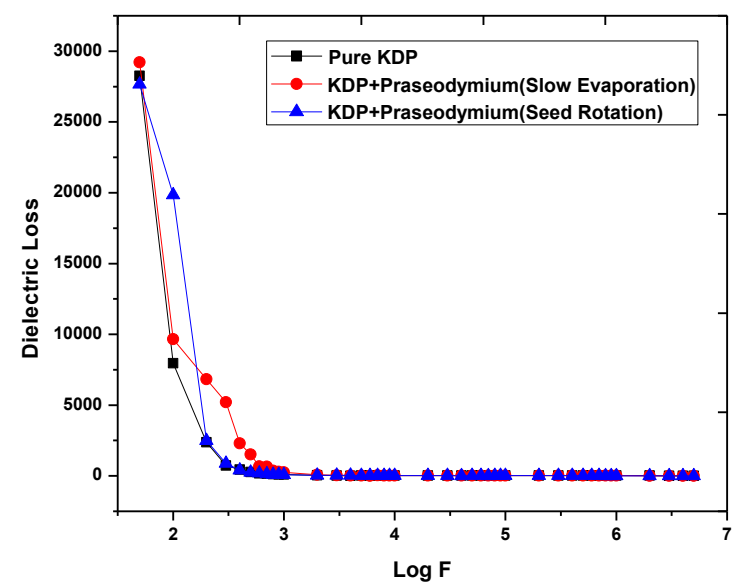

Fig. 10:- Variation of dielectric Loss with log frequency for grown crystals

As frequency increases one polarization mechanism after another is frozen out. The first to stop contribution to $\varepsilon$ is orientational component, then the ionic and lastly the electronic. The dielectric loss $(\tan \delta)$ is due to the resistive component that makes them to decrease, so that they dissipate some of the applied ac energy. Tan $\delta$ in the present study was found initially to decrease with frequency and later almost a constant over a range of frequencies. 
It is seen that a.c. conductivity is governed by the presence of a small number of free charges which result in small leakage or conduction currents and by the displacement of bound charges that give rise to polarization or displacement currents in the solid state dielectrics whereas in the d.c. conductivity there is no contribution from localized charges.

Further ionic conduction plays a major role at higher temperatures for both a.c and d.c conductivity because at higher temperatures some ions detach from the sites of crystal lattices [14]. At low frequency space charge polarization is dominant mechanism in the transport processes which is absent at high frequencies. The space charge polarization decreases with increase in frequency due to inertia of the ions to follow the variation in field.

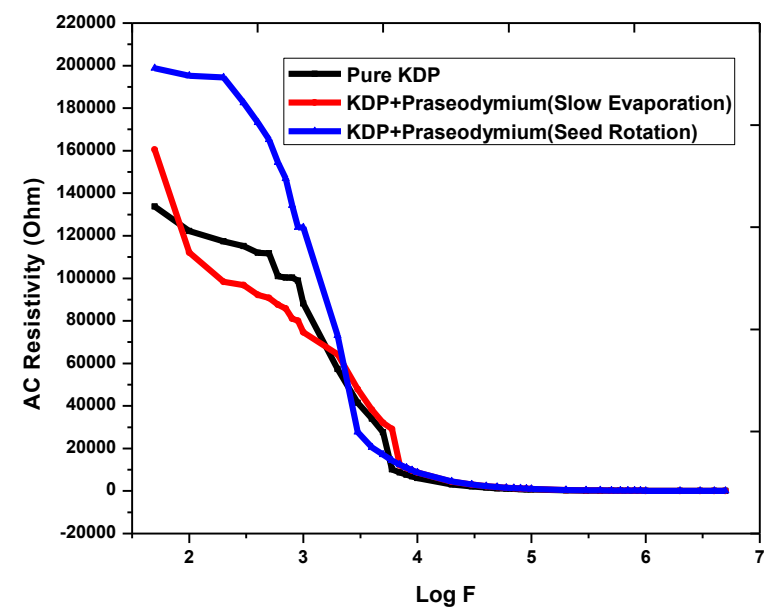

Fig 12:- Variation of AC resistivity with log frequency for grown crystals

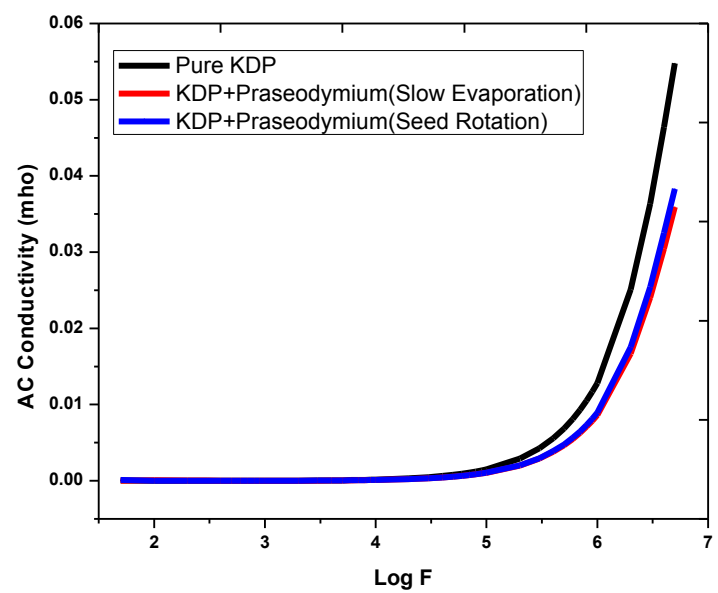

Fig 12:- Variation of AC Conductivity with log frequency for grown crystals

The variation in resistivity and conductivity with the frequency for the grown crystals are shown in Fig. 12 \& 13 . The a.c resistivity and a.c conductivity were calculated using the relation :

$$
\begin{aligned}
& \rho=\frac{A}{2 \pi \mathrm{fCd}} \\
& \sigma \rho=\frac{1}{\rho}
\end{aligned}
$$


Where $\mathrm{C}$ is the capacitance, $\mathrm{d}$ is the thickness, $\mathrm{A}$ is the area of the crystal and $\mathrm{f}$ is the frequency of the applied field. It is observed that a.c resistivity decreases rapidly as frequency increases. Obviously reverse trend was observed for a.c conductivity of the grown crystals which is considered to be a normal dielectric behavior [15].

\section{SHG studies:-}

The Second harmonic generation efficiency was determined by Kurtz powder technique [16]. Laser beam coming from the source has very high energy. Its intensity is reduced by using glass plates and Neutral density (ND) filter which reduces the intensity and it allows only $1064 \mathrm{~nm}$ wavelength to incident on the sample taken in a micro capillary tube. Output from the sample is passed through the monochromator which is intensified by photomultiplier tube and finally the signal is observed and read on the Oscilloscope. A Q-switched Nd:YAG laser beam of wavelength $1064 \mathrm{~nm}$ and $8 \mathrm{~ns}$ pulse width with an input rate of $10 \mathrm{~Hz}$ was used to test the NLO property of the sample. The second harmonic signal of $532 \mathrm{~nm}$ green light was collected by a photomultiplier tube . The optical signal incident on the PMT was converted into voltage output at the cathode ray oscilloscope.

The grown crystals were crushed into fine powder and tightly packed in a micro capillary tube. It was mounted in the path of Nd-YAG laser beam of energy $5 \mathrm{~mJ} / \mathrm{pulse}$. The KDP crystal was used as a reference material. The transmitted beam voltage for pure KDP crystal was $4 \mathrm{mV}$, for the Praseodymium doped KDP (Slow Evaporation method) crystal was $4.19 \mathrm{mV}$, Praseodymium doped KDP (Seed Rotation method) crystal was $4.89 \mathrm{mV}$ respectively. It is found that the SHG efficiency of the praseodymium doped KDP (Seed Rotation method) crystal is 1.22 times greater than KDP and praseodymium doped KDP (Slow Evaporation method) crystal is 1.04 times greater than KDP. The measured values are given in Table 6. Output intensity of SHG gives relative values of NLO efficiency of the material. The relative SHG efficiency of the grown crystals is higher than that of KDP sample which indicates the suitability of crystals for application in nonlinear optical devices and optoelectronic devices. The increased SHG efficiency is due to higher polarizability of the material than that of KDP.

Table 6:- SHG Signal and SHG efficiency of grown crystals.

\begin{tabular}{|c|c|c|}
\hline Details of the Sample & SHG Signal & SHG Efficiency w.r.t KDP \\
\hline Pure KDP & $4.00 \mathrm{mV}$ & 1.00 \\
\hline KDP doped Praseodymium(Slow Evaporation) & $4.19 \mathrm{mV}$ & 1.04 \\
\hline KDP doped Praseodymium (Seed Rotation) & $4.89 \mathrm{mV}$ & 1.22 \\
\hline
\end{tabular}

\section{Conclusions:-}

A new additive rare earth Praseodymium was added to KDP and crystals were grown by slow cooling method using microcontroller based seed rotation technique. Powder XRD and EDAX analysis confirm the fact that the Praseodymium has gone into the lattice sites of the KDP crystals. The presence of additional peaks in the XRD spectrum of doped KDP crystals shows the presence of additional phases due to doping. The presence of various functional groups was confirmed by FT-Raman spectrum. The UV-Vis transmission spectra show a wide transparency window without any absorption. KDP doped praseodymium crystals generate optical second harmonic frequency of an Nd:YAG laser. The Kurtz powder technique indicates that the SHG efficiency of praseodymium doped KDP (Seed Rotation method) crystal is 1.22 times greater than KDP and praseodymium doped KDP (Slow Evaporation method) crystal is 1.04 times greater than KDP, which indicates the suitability of crystals for application in nonlinear optical devices and optoelectronic devices. The dielectric studies show that the grown crystal has low dielectric constant and low dielectric loss.As the crystal has wide transparency in the UV and visible regions, low dielectric constant and dielectric loss, implies that this crystal can be used as a potential material for optical applications.

\section{Acknowledgments:-}

The scientific supports extended by Department of IPC for SHG studies, Department of physics for Xrd analysis, Department of materials Engineering for UV-Visible analysis, IISC, Bangalore are gratefully acknowledged. Author would like to thank DSTPURSE program for providing the Raman Spectroscopy measurement facility at Department of Physics, Bangalore University, Bangalore.

\section{References:-}

1. A..Yokotani., T. Sasaki., K.Yamanaka, C.Yamanaka, Appl. Phys. Lett. , 48 (1986) 1030.

2. S.SenGupta,T.Kar,S.P.Sen Gupta,Mater.Chem.Phys. 58(1999) 227. 
3. D. Xu. D. Xue, J. RareEarth 24(2006) 228.

4. L. N. Rashkovich, KDP Family of Single Crystals, Adam Hilger, New York, (1991).

5. J. W. Mullin, Crystallization, third ed., Butterworth Heinemann, London, (1993).

6. K. Srinivasan, K. Meera, P. Ramasamy, Cryst. Res. Technol., 35 (2000) 291.

7. M. Jayaprakasan, N.P. Rajesh, V. Kannan, R. Bairava Ganesh, G. Bhagavannarayana, P. Ramasamy, Mater. Lett., 61 (2007) 2419.

8. G. Li, X. Liping, G. Su, X. Zhuang, Z. Li, Y. He, J. Cryst. Growth, 274 (2005) 555.

9. P.V.Dhanaraj, N.P. Rajesh, C.K. Mahadevan, G. Bhagavannarayana, Physica B, 404 (2009) 2503-2508

10. M. Nakatsuka, K.Fujioka, T. Kanabe, H. Fujita, J.Gyst. Growth, 171(1997) 531.

11. G. W. Lu, and X. Sun, Cryst. Res. Technol., 37 (2002) 93.

12. B. W. Batterman and H. Cole, Rev. Mod. Phys., 36 (1964) 681

13. K. Balasubramanian , P. Selvarajan and E. Kumar, Indian Journal of Science and Technology, (2010) Vol. 3 No.1.

14. R. Priya, G. Bhagavannarayana, S. Krishnan and S. Jerome Das, Archives of Applied Science Research, 2 (4) (2010) 111-118.

15. S.Suresh, A. Ramanand, P. Mani and K. Anand, Archives of Applied Science Research, 2 (4) (2010) 119-127.

16. S.K.Kurtz, T.T.Perry, J.Appl. Phys.., 39 (1968) 3798. 